To appear in University of Massachusetts Occasional Papers in Linguistics 18: Papers in Optimality Theory, edited by Jill Beckman, Suzanne Urbanczyk, and Laura Walsh.

\title{
Object Agreement in Palauan: Specificity, Humanness, Economy and Optimality ${ }^{*}$
}

\author{
Ellen Woolford \\ University of Massachusetts
}

Palauan exhibits a complex pattern of object agreement, conditioned by aspect, specificity, number, and humanness (Wilson 1972, Josephs 1975, Georgopoulos 1991, 1992). While current syntactic theory provides different structural positions for objects that trigger object agreement and objects that do not (e.g. Mahajan 1990, Georgopoulos 1992), it does not yet provide a complete answer to the question of which objects will occupy which of these positions and why, especially in a pattern of agreement as complex as the one in Palauan. For Palauan, the following three questions need to be answered:

(1) Why is such a peculiar disjunction of features associated with object agreement in Palauan? [+human] and/or [+specific and +singular]

(2) Why are exactly the same features associated with inserted prepositions, but not base generated prepositions?

(3) Why does object agreement only occur in the perfective aspect, while preposition insertion is limited to imperfectives?

Questions of this sort have usually been assumed to involve theoretically uninteresting language specific conditions. The goal of this paper is to answer these questions in a more interesting way, making maximal use of universal principles and minimizing the need for language specific stipulations. Although this paper focuses on Palauan, the solution developed for this language has wider

\footnotetext{
*I would like to thank Kyle Johnson, Angelika Kratzer, John McCarthy, Margaret Speas, and the members of my fall 1994 syntax seminar at the University of Massachusetts for helpful comments and discussion of the material in this paper.
} 
theoretical implications, involving claims about the kinds of principles that are available in UG, how those principles are formulated, how they interact with each other, and how language specific information is encoded.

The basic idea of the account proposed here is that economy principles keep objects in their base positions, unless that would violate exclusion principles. Exclusion principles (modeled on the work of Diesing 1992 and Diesing and Jelinek 1993) exclude NPs with certain features from object positions inside VP. It is argued here that there a family of such exclusion principles available in UG, based on a different semantic features including, not only specificity, but also humanness, animacy, and number. In Palauan, we find a principle that excludes human NPs from object positions and a separate principle that excludes singular, specific NPs from object positions. The operation of these two exclusion principles is what produces the disjunction of features associated with object agreement in Palauan (question one above). NPs with the features referred to by either exclusion rule are forced out of the VP (in perfective clauses), moving to Spec Agr-O where they trigger object agreement.

However, movement out of the VP is not the only option available to avoid a violation of one of the exclusion principles. Objects can also undergo preposition insertion. We see this strategy operating in Palauan imperfective constructions, as well as in Spanish, where specific human direct objects are marked with $a$. The answer to the question of why $\mathrm{P}$ Insertion and object agreement are associated with exactly the same features in Palauan is that preposition insertion and movement to Spec Agr-O are two alternate strategies for dealing with the same problem: removing objects from positions in which they violate exclusion principles.

This leaves the third question: why is the strategy of movement to Spec Agr-O limited to the perfective aspect while preposition insertion is limited to imperfectives in Palauan. It will be shown here that using only conventional syntactic mechanisms to deal with this aspectual difference produces only a stipulative and redundant account for the Palauan facts. Since we find the language internal difference in the two aspects of Palauan paralleled in the difference between Swahili (which only allows the movement strategy) and Spanish (which only allows the preposition insertion strategy), it is reasonable to use the same mechanism to encode the Palauan-internal difference as we would use to code the cross-linguistic difference between Spanish and Swahili. Within the Optimality Theory of Prince and Smolensky 1993, cross-linguistic differences are dealt with in terms of differences principle ranking and there have been several proposals to characterize language internal differences in terms of the reranking of one principle in a particular domain (Kisseberth 1993, Rosenthall 1994, Cohn and McCarthy 1994, and Itô and Mester 1995). This approach works extremely well for the aspectual differences in Palauan. All of the differences between perfective and imperfective constructions in Palauan (including unexpected differences in the behavior of double object constructions not 
mentioned above) fall out simply and completely if the rank of one of the economy principles changes in the perfective aspect.

Treating aspectual differences in the syntax of clauses in terms of principle re-ranking allows us to account for the fact that aspect can have radical effects that are not local with respect to the aspect node. In Palauan, aspect determines whether or not $\mathrm{P}$ insertion is available to objects and it is well known that the Case pattern of entire clauses can be determined by aspect in split ergative languages, e.g. Hindi (Mahajan 1990).

This paper is organized as follows. Section one summarizes the data and generalizations about object agreement and preposition insertion in Palauan from Wilson 1972, Josephs 1976, and Georgopoulos 1991, 1992. Section two establishes the foundation for a syntactic account of these facts, associating the different types of objects in Palauan with different syntactic positions, based on proposals in Mahajan 1990, Georgopoulos 1992 and Chomsky 1992. Section three takes up the question of how to deal with the particular semantic features associated with object agreement and $\mathrm{P}$ Insertion and demonstrates why an approach that associates different semantic features/interpretations with different object Cases (e.g. de Hoop 1989, 1992) is inadequate for a pattern as complex as the one in Palauan.

Section four introduces Diesing's 1992 Mapping Hypothesis and shows that Diesing's approach requires the addition of an economy principle (Avoid Movement) in order to work as intended, but that once this economy principle is added, the portion of the Mapping hypothesis that refers to the features of NPs outside the VP becomes redundant; that is, it becomes possible to simplify Diesing's approach to refer only to the features required of NPs inside the VP. When we add the Palauan facts to this discussion, we find that the operation of two different mapping/exclusion principles in one language would create insoluble conflicts if we did not simplify Diesing's approach in this way. The simplified and modified version of Diesing's approach developed here takes the form of exclusion principles that exclude NPs with certain features from object positions inside VP.

A second economy principle, Avoid Insertion, is added in section five, motivated by the fact that preposition insertion is only used in Palauan to avoid violating one of the exclusion principles. An informal picture is presented in this section of how the two economy principles interact with the two exclusion principles to produce the Palauan data in section one. This section ends with a brief discussion of the role of similar exclusion principles in Swahili and Spanish. The problem of accounting for the striking differences in perfective and imperfective constructions in Palauan is taken up in section six. An approach limited to conventional syntactic mechanisms is compared with an Optimality approach involving the re-ranking of one principles in the perfective aspect. Section seven summarizes the proposed OT account. 


\section{Data and Generalizations}

This section lays out the data and generalizations about object agreement and preposition insertion in Palauan established in Josephs 1975 and Georgopoulos 1991, 1992. We will see that "direct objects occur as objects of a preposition in imperfective clauses under the same conditions that trigger object agreement in the perfective (Georgopoulos 1991, p. 29)". These conditions are as follows: objects must be either [+human] and/or [+specific and +singular] to trigger object agreement or preposition insertion (Josephs 1975, Georgopoulos 1991). ${ }^{1}$ Objects without these particular features are unmarked.

Examples with only one object will be considered first. These are organized into subsections based on aspect. Within each aspect, examples involving non-human objects will be presented first, followed by examples with human objects. Following this, the additional complications of double objects are discussed. Second objects in perfective examples are exempt from the conditions on other objects, yet in imperfectives, second objects conform to these conditions.

\subsection{Perfective Aspect and Object Agreement}

In the perfective aspect, direct objects trigger object agreement if they are human. Non-human objects may also trigger object agreement, but only if they are both specific and singular.

\subsubsection{Non-human objects}

In order for a non-human object to trigger object agreement, it must be both specific and singular, as in (4a). For non-specific or plural objects, there is no object agreement, as shown in (4b):

\footnotetext{
(i) Ak-milnguiu er a buk er sei er a lemei a Moses. R-1s-read P book P when P IR-come Moses I was reading a book when Moses came.

(ii) Ak-mil'er-ar a buk er a Rose. R-1s-PF-buy-3s book P Rose I bought a book for Rose.
}

${ }^{1}$ Georgopoulos 1991 describes the relevant feature for object agreement and $\mathrm{P}$ insertion as "very loosely speaking, definite (p. 24)". Josephs 1975 uses the term "specific". Since all definites are also specific, we have to look at examples with indefinite objects to determine whether definiteness or specificity is being marked in Palauan. Although Palauan does not mark NPs with definite and indefinite articles, if the article in the English glosses is a reliable indication of the (in)definiteness of Palauan objects, the relevant semantic notion is not definiteness. In the examples below, the object is translated with an indefinite article, yet the Palauan version of the sentence marks the object with a preposition or with object agreement. 
(4)

a. Te-'illebed-ii a bilis a rengalek. 3p-Pf-hit-3s dog children

The kids hit the dog.

b. Te-'illebed a bilis a rengalek.

3p-Pf-hit dog children

The kids hit a dog/the dogs/some $\operatorname{dog}(\mathrm{s})$. (Georgopoulos 1991, p. 30)

\subsubsection{Human objects}

When the object is human, it triggers object agreement regardless of what other features it has. In the following examples, we see that the human object triggers object agreement even when it is plural.

a. Mchelebed-ii a ngalek!

hit-3s child

Hit the child!

b. Mchelebede-terir a rengalek!

hit-3p children

Hit the children! (Josephs 1975, p. 395)

(6) a. Ak milsa a Droteo er a party.

I saw-3s Droteo at party.

I saw Droteo at the party. (Josephs 1975 p. 324)

b. Ak mils-terir a retede el sensei.

I saw-3p three teacher

I saw three teachers. (Josephs 1975, p. 43)

\subsection{Imperfective Aspect and P Insertion}

In the imperfect aspect, there is no object agreement. Instead direct objects are marked by a preposition if they have the features [+human] or [+specific, +singular] that are associated with object agreement in the perfective aspect. Objects without these features remain unmarked, as in the perfective aspect.

\subsubsection{Non-human objects}

Non-human objects require $\mathrm{P}$ Insertion in imperfectives if they are both specific and singular, as in the (a) examples below. If they are non-specific and/or plural, no P Insertion occurs, as shown in the (b) examples.

(7) a. Ng-milengelebed er a bilis.

3s-Im-hit P dog

S/he hit the dog. 
b. Ng-milengelebed a bilis.

3s-Im-hit dog

S/he hit a dog /the dogs /some dogs. (Georgopoulos 1991, p. 29)

a. Ak ousbech er a bilas er a klukuk.

$I$ need $P$ boat tomorrow

I need the boat tomorrow.

b. Ak ousbech a bilas er a klukuk.

I need boat tomorrow

I need a boat / the boats tomorrow. (Josephs 1975, p. 49)

\subsubsection{Human objects}

Human objects always require $\mathrm{P}$ Insertion in the imperfective, even when the object is plural.

(9) A sensei a mengelebed er a rengalek.

teacher R-hit $\mathrm{P}$ children

The teacher is hitting the children. (Georgopoulos 1991, p. 35)

\subsection{Double Object Constructions}

Although double object constructions are not common in Palauan, they do occur. Georgopoulos 1992 discusses perfective examples involving the verb 'give'. In such constructions, only the goal triggers object agreement, never the theme (Georgopoulos 1992, p. 168).

(10) Ng-mils-terir a buu' a rengalek a Sabino.

3s-gave-3p betel nut children Sabino

Sabino gave the kids some betel nuts. (Georgopoulos 1992, p. 168)

(11) Ak-mils-terir a buk.

1s-gave-3p book

I gave them a/the book. (Georgopoulos 1992, p. 168)

What is interesting is that the theme, which does not have the option of triggering object agreement, is not restricted to the semantic features usually associated with an unmarked object. Instead, it can be interpreted as a singular specific, as in (11), a reading that is ordinarily limited to objects triggering agreement. Thus, the behavior of a theme as a second object contrasts markedly with the behavior of a theme as a sole object, but only in perfective constructions. In imperfective constructions, the second object is treated just like the first object; if it is human and/or specific and singular, it is marked with a preposition. In the example below, both objects have the semantic features associated with preposition 
insertion (one is human and the other is singular specific) and both objects are marked with prepositions.

(12) A Romana a omeka er a rengalek er a kukau.

Romana feed $\mathrm{P}$ children $\mathrm{P}$ taro

Romana is feeding the children the taro. (Josephs 1975, p. 207)

1.4 Restriction of Syntactic Devices by Aspect

In the examples above, object agreement occurs only in the perfective aspect, while preposition insertion occurs only in imperfectives. This restriction by aspect of these two syntactic devices is strictly upheld in Palauan, according to Josephs 1975 . Even in an example like $(13,53)$ below, where only one object can trigger object agreement, presumably because there is only one structural position available for object agreement (Georgopoulos 1992), the second object cannot be marked by $\mathrm{P}$ insertion even when it is singular and specific. "There is no overt way of marking this [second] object as specific vs. non-specific" (Josephs 1975, p. 347).

(13) Ak-mils-terir a resechelik a hong. $1 \mathrm{~s}-$ gave- $3 \mathrm{p}$ friends book I gave my friends a/the book. (Josephs 1975, p. 347)

\subsection{Summary}

The generalizations concerning object marking in Palauan can be summarized follows:

a. Perfective clauses

The thematically highest object triggers object agreement if it is [+human] and/or [+specific and +singular].

Preposition insertion is not possible.

b. Imperfective clauses

Objects are marked with inserted prepositions if they are [+human] and/or [+specific and +singular].

Object agreement is not possible.

The remaining sections address the question of how to account for these generalizations. 


\section{Object Positions in Syntax}

The first step in developing an account of the above generalizations is to determine how much of this problem can be solved using the devices available in current theory. The current structural approach to object Case and agreement (e.g. Mahajan 1990, Chomsky 1992, Georgopoulos 1992) provides a foundation for an account of the Palauan data by providing different syntactic positions for objects that trigger agreement and those that do not. The structures proposed here for Palauan are identical to those proposed by Georgopoulos 1992, except for the position of agreeing objects. For reasons that will become clear in section three, it is necessary to follow Mahajan 1990 and Chomsky 1992 in assuming that objects that trigger object agreement move out of the VP, to Spec Agr-O, while objects that do not trigger agreement remain in their base positions in the VP as in Georgopoulos 1992.

The proposed structure for perfective clauses with object agreement, as in example (1a), repeated below, is shown in (16): ${ }^{2}$

(15) Te-'illebed-ii a bilis a rengalek.

3p-Pf-hit-3s dog children

The kids hit the dog.

$\begin{array}{llll}\mathrm{NP}_{\mathrm{i}} & & & \\ \operatorname{dog} & & & \\ \text { Agr-O } & & \mathrm{VP} & \\ & & & \\ & & & \\ & \mathrm{V} & & \mathrm{NP}_{\mathrm{i}} \\ & \text { hit } & \end{array}$

\footnotetext{
${ }^{2}$ These trees do not accurately reflect the surface word order of verb object. This surface order may be accounted for in one of several possible ways and the decision is not crucial for this paper. For example, the verb may raise to Tense or Agr-S or the object may not move until LF.
} 
When the object does not agree, the structure is as shown in (18):

(17) Te-'illebed a bilis a rengalek.

3p-Pf-hit dog children

The kids hit a dog/the dogs/some dog(s). (Georgopoulos 1991, p. 30)

$$
\begin{aligned}
& \text { NP } \\
& \text { children }
\end{aligned}
$$

\section{Agr-S}

$\begin{array}{crr}\text { Agr-O } & & \text { VP } \\ & & \\ \text { V } & & \text { NP } \\ \text { hit } & \text { dog }\end{array}$

Now let us turn to the structure of clauses in the imperfect aspect, where there is no object agreement. Construction in which the object remains unmarked in its base position, as in (19a), have the same structure that we saw in (18) above. Objects in examples like (19b) are inside a PP, as in (20): ${ }^{3}$

a. Ng-milengelebed a bilis.

3s-IM-hit dog

S/he hit a dog/the dogs/some dogs.

b. Ng-milengelebed er a bilis.

3s-Im-hit P dog

S/he hit the dog. (Georgopoulos 1991, p. 29)

\footnotetext{
${ }^{3}$ It is not necessary to assume that this PP moves out of the VP under the analysis developed below.
} 


$\begin{array}{llll}\text { Agr-S } & & \text { NP } \\ \text { Agr-O } & & & \\ & & \text { VP } & \\ & & & \\ & \text { V } & & \text { PP } \\ & \text { hit } & & \\ & & \text { P } & \text { NP } \\ & & \text { er } & \text { dog }\end{array}$

In the analysis to be developed below, it is assumed that the preposition in this construction is inserted. The syntactic device of preposition insertion has been a fairly standard one in the theory since Chomsky 1981. Of Insertion accounts for the fact that the object of a verb in a nominalized construction, such as 'the destruction of the city', is marked with a preposition, despite the fact that the basic verb 'destroy' is not subcategorized to take a PP, as we see from VPs like 'destroy the city'. Similar circumstances occur in Palauan. We see from the behavior of verbs such as 'hit' in perfective clauses that they are not subcategorized to take PP objects, yet in the imperfective, objects of those verbs with certain features are marked with a preposition, as we saw in section one.

Palauan also has base generated PPs, which do not require any special features of their objects. In (21a), we see a plural object in a PP, in contrast to the example above in (19), where a plural object cannot be marked with an inserted preposition:

a. Ak-mo er a katsudo. 1s-go $\mathrm{P}$ movies I am going to the movies. (Georgopoulos 1991, p. 26)

b. Aki-mle kaudenge er a Kuam. 1p-Past Recip-know P Guam We knew each other in Guam. (Georgopoulos 1991, p. 55, from Josephs 1975, p. 124)

However, the preposition that is inserted, er, is identical in form to the preposition that is base generated, er, and it is assumed here, following Georgopoulos 1991, that the same preposition, er, occurs in both constructions (and, in fact, Palauan only has one preposition). In the account presented in section five, the difference in the derivational history of the preposition (inserted versus base-generated) is sufficient to account for the lack of any semantic features associated with the 
objects of base generated prepositions, and the fact that the semantic features associated with the objects of inserted prepositions are identical to those associated with object agreement. It is not necessary to adopt Joseph's 1975 view that Palauan has two different prepositions that happen to look alike.

Establishing different structural positions for the different types of Palauan objects is only the first step toward an analysis of the data in section one, however. We still need to know what determines which position a particular object will occupy.

\section{An Attempt to Associate Features with Cases}

The next task we face is how to force NP Movement to Spec Agr-O (or preposition insertion) to occur just when the object has the particular features [+human] and/or [+specific and +singular]. Since Case is assumed to drive NP Movement and to motivate preposition insertion (Chomsky 1981), an obvious way to approach this problem is to propose that the Case that is assigned to objects in their base position in the VP is only available to objects with particular features. A proposal along these lines is developed for Turkish by de Hoop 1989, 1992.

In Turkish, specific objects are marked with an overt Case morpheme, while non-specific objects have no overt Case morphology. De Hoop 1989, 1992 proposes that in Turkish, and universally, there are two different Cases for objects. One object Case, strong Case, only marks objects that get interpreted as generalized quantifiers. Thus specific objects get strong Case, while non-specific objects get weak Case. Although de Hoop assumes that both of these object Cases are assigned by the verb inside the VP, it would be a small modification to propose that the Case associated with specific objects is assigned by Agr-O (in order to account for the association of specific objects and object agreement in languages like Palauan). Under such an account of Turkish, a non-specific object would receive Case in its base position from the verb while a specific object would move to Spec Agr-O for Case from Agr-O. A specific object could not remain in its base position, nor could a non-specific object move to Spec Agr-O, because the Cases available in those positions would require different features.

Let us attempt to develop an approach of this type for the Palauan facts discussed in section one. To do this, we will need to the associate features with each of the different objects Cases. The Case assigned by $\mathrm{V}$ to objects in their base position will have to require the features [-human] and either [-specific] or [-singular]. The Case assigned by Agr-O will require the features [+human] and/or [+specific and +singular]. To capture the fact that exactly the same features are required by inserted prepositions, we will have to assume that inserted prepositions assign the same Case that Agr-O does. Since base generated prepositions are not associated with any particular semantic features, we will have to follow Josephs 
1975 in assuming that these are different prepositions, assigning different Cases, even though they look alike.

(22) Summary of Features on Cases Approach to Palauan

a. Case assigned by $\mathrm{V}$ : requires [-human] and

[-specific] and/or [-singular]

b. Case assigned by Agr-O: requires [+human]

and/or [+specific and +singular]

c. Case assigned by inserted prepositions: same as b.

d. Case assigned by base generated prepositions: requires no special features

As formulated above, however, this approach misses an important generalization: the features associated with the Case assigned by $\mathrm{V}$ are exactly the opposite of the features associated with the Case assigned by Agr-O and inserted prepositions. If these features are specified independently, this generalization should not hold. However, we can modify this approach in a way that captures this generalization, and also simplifies the account and eliminates the need to claim that base generated and inserted prepositions are different prepositions that assign different Cases.

In the modified version of the 'features on Cases' approach to Palauan, we eliminate all of the feature specifications associated with Cases except those associated with the Case assigned by V. The model will then work as follows. If an object has the features necessary to get Case from $\mathrm{V}$, that object will get that Case and remain in its base position. Only objects without the features required by $\mathrm{V}$ will be able to move to Spec Agr-O (or undergo P Insertion). As a result, objects in Spec Agr-O (or inserted PPs) will have features opposite of those required by $\mathrm{V}$, but by default, not because of any feature requirements of Agr-O or inserted P. Since inserted prepositions have no feature requirements under this approach, they can be considered to be the same preposition, assigning the same Case, as a base generated preposition.

a.

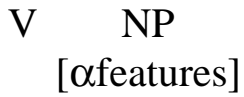

b.

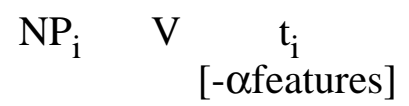

To insure that objects that can get Case from $\mathrm{V}$ do not move or undergo $\mathrm{P}$ Insertion, we cannot rely on the feature clash approach outlined above, since Agr-O and inserted $\mathrm{P}$ have no feature requirements under this modification. Instead, we could specify that Case assignment by $\mathrm{V}$ is obligatory, if possible. 
Alternatively, we could appeal to economy principles along the lines of Chomsky 1989 to prevent unnecessary movement or insertion.

Modified Features on Cases Approach to Palauan

a. Case assigned by $\mathrm{V}$ : requires [-human] and

[-specific] and/or [-singular]

b. Case assigned by Agr-O: requires no special features

c. Case assigned by prepositions: requires no special features

While this modified version of the 'Features on Cases' approach has the advantage of providing a simple and appealing answer to the second question posed in the introduction to this paper (why exactly the same features are required for object agreement as for preposition insertion, but not for base generated prepositions), this account cannot deal with the behavior of double object constructions without additional ad hoc stipulations. Recall from section one that the second object in a perfective double object construction remains unmarked in its base position regardless of what features it has:

Ak-mils-terir a resechelik a hong.

1s-gave-3p friends book

I gave my friends a/the book. (Josephs 1975, p. 347)

We can explain why the second object cannot trigger object agreement by assuming that there is only one Spec Agr-O position available. Moreover, we can appeal to some principle such as minimality to prevent the second object from moving across the first object to Spec Agr-O. However, we would be left with the problem of what Case the second object gets in its base position. If it gets the Case assigned by V, it should be limited to the features compatible with that Case: [-human, -specific] and/or [-human, -singular]. But the second object in a perfective construction is not limited to those features. Therefore, one would have to propose that there is a different Case available for second objects, a Case with no feature requirements. While the idea of different Cases for the two objects in a double object construction is used in work such as Burzio 1986, proposing a different Case for the second object creates more problems than it solves in Palauan. One would have to explain why verbs are not capable of assigning the same Case to second objects in imperfective constructions. As we saw in section one, the second object in an imperfective construction is not free to have just any features. Instead, if it does not have the features required for the Case assigned to first objects, it must undergo P Insertion.

A Romana a omeka er a rengalek er a kukau.

Romana feed $\mathrm{P}$ children $\mathrm{P}$ taro

Romana is feeding the children the taro. (Josephs 1975, p. 207) 
A second problem with the proposal that verbs can assign two different Cases in Palauan is how to prevent the special Case for second objects from being assigned to first or single objects. One could not solve this problem by claiming that the special Case for second objects is an inherent Case limited to themes, because single objects are often themes. It is not clear how one could avoid simply stipulating that this special Case is only available to the second object in perfective clauses.

In addition to these empirical problems, there are conceptual problems with a 'features on Cases' approach to Palauan. The modifications to de Hoop's approach that are required to make it work for Palauan undermine all of the attractive aspects of that approach. De Hoop 1989, 1992 proposed that the two object Cases proposed for Turkish are actually universal and there is a universal mapping from Case to semantic interpretation. But in Palauan, the particular Cases proposed for Turkish would play no role at all. Instead, the semantic features associated with the two object Cases would have to be quite different. Instead of simply associating the weak Case with non-specific objects as in Turkish, the weak Case in Palauan would require the object to be [-human] and either [specific] or [-singular]. The simple idea that NPs with strong Case get interpreted as generalized quantifiers would not work for Palauan since the strong Case would have to require the features [+human] and/or [+specific and +singular]. No sort of features on Cases approach can deal easily with the choice of features that would have to be associated with Cases in Palauan.

Thus a 'features on Case' approach provides only an awkward and stipulative account of the Palauan facts. The following sections develop an alternative approach to these Palauan facts, building on work such as Diesing 1992 and Diesing and Jelinek 1993. However, this alternate approach will preserve the appealing portion of the modified 'features on Case' account developed above, which answers the question of why exactly the same features are required for object agreement as for preposition insertion (but not base-generated prepositions.

\section{Specificity and Humanness Mapping}

Since specificity plays an important role in Palauan object marking, it makes sense to explore the possibility that Diesing's 1992 Mapping Hypothesis, which excludes specific NPs from object positions in the VP, is operating in Palauan. Although only [+singular] NPs show specificity effects in Palauan, Diesing and Jelinek 1993 and Comrie 1981 suggest that what counts as specific may vary from language to language.

In this section, it will be argued that a modified version of Diesing's principle is what accounts for the fact that [+specific, +singular] objects trigger object agreement (or P Insertion) in Palauan. To account for the parallel behavior of human objects, it is proposed that there is a similar principle operating in 
Palauan, based on humanness instead of specificity. That principle excludes [+human] NPs from object positions inside the VP. Thus the odd disjunction of features associated with marked objects in Palauan, [+specific, +singular] and/or [+human] is accounted for here by the operation of two similar, but distinct principles. The features associated with unmarked objects that remain in the VP, [-human, -specific] and/or [-human, -singular], is simply the features that do not violate either of these principles.

The organization of this section is as follows. Diesing's 1992 proposal is introduced in section 4.1 and applied to Palauan. The proposal for a parallel treatment of [+human] NPs is developed in section 4.2. Section 4.3 focuses on a conflict that arises in trying to satisfy both specificity mapping and humanness mapping at once. This conflict is resolved by simplifying these mapping principles so that they continue to exclude NPs with certain features from inside the VP, but say nothing about the features of NPs outside the VP. Arguments are presented in section 4.4 that this modification of Diesing's proposal accounts for the same range of syntactic facts that the original proposal does.

\subsection{Diesing's Mapping Hypothesis}

It is well known that in languages like Icelandic, specific objects can undergo Object Shift while non-specific objects cannot. In (27), we see that a specific object optionally shifts leftward, from its position inside the VP in (27a), across the negative (which is outside the VP), in (27b). In (28), we see that a nonspecific object cannot undergo object shift. Only the (a) version, in which the object remains inside the VP, is grammatical.

a. Hann las ekki bækurnar. he read not books-the

He did not read the books.

b. Hann las bækurnar ekki. he read books-the not

He did not read the books.

a. Ég las ekki bók.

I read not book

I did not read a book.

b. *Ég las bók ekki.

I read book not

To account for facts of this sort, Diesing 1992 proposes a "Mapping Hypothesis", under which the semantic interpretation of an NP is determined by its location, inside or outside the VP: 
a. material from VP is mapped into the nuclear scope

b. material from IP is mapped into a restrictive clause

As a result of the Mapping Hypothesis, NPs inside the VP are assigned a nonspecific interpretation, while NPs outside the VP may be interpreted as specific. To account for the fact that there are exceptions to the predicted strict correlation between specificity and syntactic position in the languages of the world, Diesing proposes that this correlation holds without exception at LF and that deviations at S-Structure are repaired by LF raising or lowering. Thus, the specific object in example (1a) above, which remains in the VP at S-Structure, undergoes Object Shift at LF to position outside the VP. Similarly, subjects in Icelandic, which can be either specific or non-specific at S-Structure (as in English), undergo LF lowering at S-Structure to bring them into line with the predictions of the Mapping Hypothesis.

In Palauan, we find that singular specific NPs trigger object agreement (in the perfective aspect), suggesting that these NPs have moved out of the VP to Spec Agr-O, conforming to the predictions of the Mapping Hypothesis. The fact that only singular NPs behave as expected under Diesing's proposal could be dealt with using some version of the idea in Diesing and Jelinek 1993 and Comrie 1981 that languages vary with respect to what they count as 'specific'. ${ }^{4}$ Without getting into the details of the proposal at this point, let us tentatively assume that Diesing's Mapping Hypothesis is what excludes [+specific, +singular] NPs from object positions inside VP in Palauan.

\subsection{Humanness Mapping}

Diesing's Mapping Hypothesis says nothing about the other class of NPs that are excluded from object positions in the VP in Palauan, [+human] NPs. The behavior of human NPs is identical to that of singular, specific NPs in Palauan and there should be a similar account of the behavior of these two types of NPs.

\footnotetext{
${ }^{4}$ Although one might be tempted to argue that all specific NPs actually move to Spec Agr-O in Palauan, but that plural NPs simply trigger a null form of agreement, there are plural forms of the object agreement morphemes in Palauan, which surface with human objects. Although one could try to argue that, perhaps only non-human plurals trigger a null form of agreement (as in Lemaréchal 1993), this proposal ignores the parallelism between perfective and imperfective aspect in Palauan: Non-human plural NPs never trigger P insertion, thus it seems most reasonable to assume that non-human objects do not trigger even non-overt object agreement.
} 
Thus, if the mapping approach is the correct way to account for the behavior of specific NPs in Palauan, then the Palauan data motivates a parallel mapping principle based on humanness. ${ }^{5}$

Let us tentatively assume, then, that in addition to specificity mapping, there is also a mapping principle operating in Palauan that excludes [+human] NPs from object positions inside VP. ${ }^{6}$ The operation of these two principles together produces the disjunction of features associated with object agreement in Palauan: [+human] and/or [+singular, +specific].

\subsection{A Conflict Between Mapping Principles}

A serious problem arises if we formulate these mapping principles with two parts, following Diesing 1992, one part referring to NPs inside the VP and one part referring to NPs outside the VP:

Mapping Principles [tentative formulation]

1. Specificity Mapping (In Palauan, [-singular] -> [-specific])

a. NPs inside VP must be [-specific]

b. NPs outside VP must be [+specific]

\section{Humanness Mapping}

a. NPs inside VP must be [-human]

b. NPs outside VP must be [+human]

Situations arise in which it would be impossible to satisfy the requirements of both mapping principles at once. In examples like (31), the object is [+specific, +singular] but [-human]. In order to satisfy the Specificity Mapping Principle, this object would have to be outside the VP at LF, but in order to satisfy the Humanness Mapping Principle, this object would have to be inside the VP at LF.

(31) Te-'illebed-ii a bilis a rengalek.

3p-Pf-hit-3s dog children

The kids hit the dog.

In (32), the situation is reversed. The object is [+human], but plural (and thus would not count as +specific for Palauan. To satisfy the Specificity Mapping Principle, this object would have to be inside the VP at LF, but to satisfy the

\footnotetext{
${ }^{5}$ Note that it would not work to claim that only human NPs count as specific in Palauan. It is clear that it is not possible to combine humanness and specificity into one mapping principle for Palauan, because human objects trigger object agreement even when they are not specific and specific, singular objects trigger object agreement even when they are not human.

${ }^{6}$ Humanness is also a factor in object agreement in languages like Swahili (see section 5.4).
} 
Humanness Mapping Principle, this object would have to be outside the VP at LF. There is no way to satisfy both principles at once.

Mchelebede-terir a rengalek!

hit-3p children

Hit the children! (Josephs 1975, p. 395)

The conflict between these mapping principles can be eliminated if the formulation of the principles is simplified. The idea is to remove the restriction on the features of NPs outside the VP, leaving only the restriction on the features of NPs inside the VP. If an NP has features that violate either principle, it will be forced out of the VP. If an NP has features that violate neither principle, it can remain in its base position inside VP.

The modified version of these mapping principles will be referred to as 'exclusion' principles, since they no longer assign interpretations to NPs, but merely exclude NPs with certain features from the VP. Formulating these principles as exclusion principles also allows us to get rid of the idea that only singular NPs are specific in Palauan. Instead, the cross-linguistic variation is what appears to count as specific can be expressed as cross-linguistic variation in whether or not the Specificity Exclusion Principle refers to other features in addition to [+specific]: ${ }^{7}$

Specificity Exclusion Principle (Palauan Variant)

Exclude [+specific, +singular] NPs from positions inside VP.

\section{Humanness Exclusion Principle}

Exclude [+human] NPs from positions inside VP.

A violation of either exclusion principle will force an NP to leave the VP. If an object is singular and specific, but not human, as in (31), the object will be forced out of the VP by the Specificity Exclusion Principle. If an object is human, but not both singular and specific, as in (32), the object is forced to move because of the Humanness Exclusion Principle. In both examples, this is the result we want because the objects in both examples trigger object agreement, indicating that these objects have moved out of the VP to Spec Agr-O. Only objects whose features are not mentioned by either exclusion principle will be allowed to remain in their base positions inside VP. In sections five and six, we will see how these

\footnotetext{
${ }^{7}$ Recall from the discussion in section two that there is no way to express these principles as positive requirements on the features that an NP in the VP must have without resorting to a choice of features: [-singular] or [-specific]. Thus the preferable formulation of the specificity principle for Palauan is in terms of the features of objects that are excluded from the VP: [+specific, +singular].
} 
exclusion principles interact with economy principles to produce the range of Palauan data discussed in section one.

Given that Palauan requires such a major modification in the formulation of the mapping principles, let us now reexamine the Object Shift data that motivated the original formulation of Diesing's 1992 Mapping Hypothesis to determine whether we can, in fact, do without the (b) portion of the Mapping Hypothesis that refers to the interpretation of NPs outside the VP. When we do, we will find that the answer is yes. The (b) portion of the mapping hypothesis has to be supplemented by an economy principle to make it do the syntactic work intended; but once this economy principle is added, it removes all syntactic motivation for the (b) portion of the Mapping Hypothesis.

\subsection{Another Look at Object Shift}

The (b) portion of Diesing's Mapping Hypothesis, which specifies what kinds of interpretations NPs outside the VP can have, is intended to account for the ungrammaticality of examples like (36b), where a non-specific object moves out of the VP.

a. Hann las ekki bækurnar. he read not books-the He did not read the books.

b. Hann las bækurnar ekki. he read books-the not He did not read the books.

a. Ég las ekki bók.

I read not book

I did not read a book.

b. *Ég las bók ekki.

I read book not
a.
$\Gamma_{V P}$ non-specific object
b. *non-specific object $\left[\mathrm{VP}_{\mathrm{V}}\right.$
$\mathrm{t}$

However, recall that under Diesing's hypothesis, LF lowering is an option that is available to repair $\mathrm{S}$-Structures that do not conform to the predictions of the Mapping Hypothesis. LF lowering is motivated by the necessity of accounting for the fact that subjects can be either specific or non-specific in languages like Icelandic or English. A non-specific subject has to be lowered to a position inside the VP at LF, or else it would violate the Mapping Hypothesis. But, if LF lowering is available for subjects, it should also be available for objects in 
examples like (36b). Thus we would expect the S-Structure in (36b) to be grammatical with a non-specific reading, even though the object is outside the VP, if LF lowering is possible.

We can deal with this problem by appealing to economy principles. Chomsky 1989 proposes an economy principle that selects the derivation with the fewest steps, that is, the fewest applications of movement. Let us call this economy principle, Avoid Movement. Each step/movement in a derivation is one violation of Avoid Movement and the derivation with the fewest violations of this economy principle is preferred. ${ }^{8}$ The intuition we want to capture is that movement out of the VP is not possible when it is not necessary. It is necessary for subjects (because of the extended projection principle), but it is not necessary for objects in examples like (36) where the object is non-specific. Thus, we want the economy principles to compare the three derivations below, where a nonspecific object remains in its base position (38a), or moves out of the VP (38b), or moves out and then lowers into the VP again (38c).
a.
$\left[_{v p}\right.$ non-specific object
b. non-specific object $\left[_{v p} \quad t\right.$
c. $\quad \mathrm{t} \quad[\mathrm{vp}$ non-specific object (LF lowering)

Avoid Movement will rule out the derivation in (38c), where two movements have taken place, in favor of (38a), where no movements have taken place. Thus the undesired LF lowering is blocked by this economy principle. However, note that once we add this economy principle, we no longer need the Mapping Hypothesis to block the derivation in (38b), because this derivation, which involves one violation of Avoid Movement is ruled out in favor of (38a) which involves no violations of Avoid Movement.

To insure that the economy principle does not throw out all derivations involving movement, the features on the object in the set of derivations that are compared must be the same. The wrong result is produced if we generate the set of derivations to be compared using an object NP whose specific or non-specific interpretation is not yet determined. To see this, observe what happens if we begin with the following candidates, one with no movement, one with movement at SStructure, and the third with movement at LF.

\footnotetext{
${ }^{8}$ This is Grimshaw's 1994 reformulation of the shortest movement idea. Grimshaw's name for this principle is 'Stay'.
} 
(39)
a.
$\left[_{\mathrm{vp}} \quad\right.$ book
b. book
$\left[\begin{array}{ll}\text { vp } & \mathrm{t}\end{array}\right.$
(S-Structure Movement)
c. book
$\left[_{\mathrm{vp}} \quad \mathrm{t}\right.$
(LF Movement)

If we let the Mapping Hypothesis apply first, assigning an interpretation to each object based on its location inside or outside the VP, we produce the following result:
a.
$\left[_{\mathrm{vp}} \quad\right.$ book [non-specific]
b. book [specific] $\left[_{\mathrm{vp}} \mathrm{t}\right.$
(S-Structure Movement)
c. book [specific] $]_{\mathrm{vp}} \quad \mathrm{t}$
(LF Movement)

If the economy principle evaluates these derivations with respect to each other, it selects the derivation in (a) without any movement, and throws out both less economical derivations in (b) and (c). This is not the desired result because it would make it impossible to ever have a specific object. The situation would not improve if we allowed the economy principles to apply first. They would still eliminate the (b) and (c) derivations, leaving the Mapping Hypothesis with only the (a) derivation to assign an interpretation to.

One solution to this problem is to abandon the idea that specific/nonspecific interpretations are assigned by the Mapping Hypothesis and, instead, view the Mapping Hypothesis as a checking mechanism. We can then generate the candidate set by beginning with lexical items whose interpretation as specific or non-specific is fixed. To see how this will allow us to produce the right result for a specific object, let us examine the effect of the Mapping Hypothesis and the economy principle on the candidate structures below.
a.
[VP $\quad$ specific object
b.
[VP $\quad$ specific object
(with LF raising)
c. specific object $\left[_{\mathrm{VP}}\right.$
$\mathrm{t}$

The (a) portion of the Mapping Hypothesis that excludes specific objects from positions inside VP will rule out (41a). The remaining two derivations, which are equivalent at LF, both obey the Mapping Hypothesis. If the economy principle, Avoid Movement, is ranked lower than the Mapping Hypothesis, so that it only gets to decide between derivations that satisfy the Mapping Hypothesis, then the economy principle only gets to compare the derivations in (b) and (c), because the derivation in (a) is out of the picture. Both the (b) and (b) candidates involve one movement. They differ only in that the movement is at LF in (b) and at S- 
Structure in (c), but this is not relevant to the economy principle, Avoid Movement. Thus, both derivations involve one violation of Avoid Movement and thus both are equivalent with respect to the economy principle. As a result, both derivations will surface (unless some other principle eliminates one or the other possibility). This is the result we want in languages like Icelandic where object shift is optional (at S-Structure) for specific objects.

The conclusion we can reach based on the above demonstrations is that only the (a) portion of Diesing's 1992 Mapping Hypothesis does any syntactic work that is not duplicated by the independently necessary economy principle, Avoid Movement. Thus we can simplify the Mapping Hypothesis so that it continues to exclude specific NPs from the VP, but says nothing about NPs outside the VP, and it will continue account for the same syntactic facts. This is consistent with the conclusion in section 4.3 that the Mapping Hypothesis must be simplified in this way in order to account for the Palauan facts. Let us now return to Palauan to see how the interaction of exclusion and economy principles account for the facts described in section one.

\section{The Interaction of Exclusion and Economy Principles in Palauan}

We now have the two exclusion principles developed in section 4.3 and one economy principle, Avoid Movement. In this section, we will add one additional economy principle, Avoid Insertion, and show how the interaction of these principles produces the right results for Palauan. In this section, we will treat perfective and imperfective constructions separately, delaying until section six the question of how to deal with the differences that aspect produces:

Exclusion Principles

a. Specificity Exclusion Principle (Palauan Variant)

Exclude [+specific, +singular] NPs from positions inside VP.

b. Humanness Exclusion Principle

Exclude [+human] NPs from positions inside VP. 


\section{Economy Principles 9}

a. Avoid Movement

b. Avoid Insertion

\subsection{The Candidate Set}

For this account to work correctly, it is necessary for the grammar to compare different versions of the same structure, with and without NP movement of the object to Spec Agr-O. For Palauan, the presence of object agreement is taken as the diagnostic of movement to Spec Agr-O. For imperfective constructions, we will also need to compare versions of the same sentence with and without preposition insertion.

Since Case is what drives NP Movement, the question arises of whether all of these derivations obey the Case Filter. If not, the Case Filter would eliminate some of these derivations before the other principles even had a chance to apply. It is assumed here that, since objects can occupy all three positions at S-Structure in Palauan (sister of V, Spec Agr-O, and object of an inserted preposition), Case is potentially available in all three of these positions. To maintain the standard assumption that NP Movement must be from a Caseless position, it is assumed here that verbs optionally assign Case in Palauan (if not universally). Each D-structure thus has two or more possible S-Structures associated with it, one in which the verb assigns Case to the object in its base position and one or more in which the verb does not assign Case. In the derivation in which Case is assigned to the object, that object must remain in its base position. In the derivations where the verb does not assign Case to its object, that object either moves to Spec Agr-O or undergoes P Insertion. All of these possible derivations satisfy the Case Filter. It is the role of the exclusion principles and the economy principles to select the best one of these derivations. As noted above in section four, it is also assumed that the features of NPs are fixed throughout the candidate set.

Let us first consider perfective constructions.

\subsection{Perfective Constructions}

Putting aside the question of why $\mathrm{P}$ Insertion never occurs in perfective construction until section six, we want the grammar to select the correct outcome

\footnotetext{
${ }^{9}$ These economy principles are formulated in a very general way that does not distinguish different types of movement or insertion. Although this general formulation works for the data considered in this paper, the only movement that is actually considered is Move NP and the only insertion that is considered is preposition insertion.
} 
from two derivations, one with no movement and the other with movement to Spec Agr-O triggering object agreement:

a. The object remains in its base position:

$$
\left[\begin{array}{lll}
\mathrm{VP} & \mathrm{NP}
\end{array}\right]
$$

b. The object moves to Spec Agr-O, triggering object agreement:

$$
\mathrm{NP}_{\mathrm{i}} \quad \mathrm{Agr}-\mathrm{O} \quad\left[\begin{array}{lll}
\mathrm{VP} & \left.\mathrm{t}_{\mathrm{i}} \quad\right]
\end{array}\right.
$$

Let us first consider an example with a non-human object that is singular and specific:

(45) Te-'illebed-ii a bilis a rengalek.

3p-Pf-hit-3s dog children

The kids hit the dog. (Georgopoulos 1991, p. 30)

Thus the two candidates the grammar needs to compare are as follows:
a.
[VP $\operatorname{dog}[+$ specific, + singular $]$

b. dog [+specific, +singular $]$ Agr-O [VP $\quad$ t $]$

The (a) derivation violates the Specificity Exclusion Principle, because it involves a [+specific, +singular] NP that remains in the VP. Thus the (a) derivation is eliminated in favor of the (b) derivation, which is consistent with all of the exclusion principles. This is the desired result, since we see that this example has object agreement. To get this result, the exclusion principles must apply before Avoid Movement, or else the economy principle would simply eliminate all derivations involving movement before the exclusion principles had a chance to apply. Thus this example provides an argument for the following ranking (in perfective clauses):

Exclusion Principles \Avoid Movement

\begin{tabular}{|c|c|c|c|}
\hline \multicolumn{2}{|c|}{ Candidates } & Exclusion & Avoid \\
\hline a. & $\begin{array}{r}\text { Object in } \\
\text { Base Position }\end{array}$ & $* !$ & \\
\hline b. & $\begin{array}{l}\text { Object Moves } \\
\text { to Spec Agr-O }\end{array}$ & & $*$ \\
\hline
\end{tabular}

(Object is [+singular, +specific, -human]) 
We get the same result in examples where the object is [+human].

(48) Mchelebede-terir a rengalek!

hit-3p children

Hit the children! (Josephs 1975, p. 395)

The two candidates the grammar needs to compare are as follows:
a. [VP children [+human, -singular] ]
b. children [+human, -singular] Agr-O [ ${ }_{\mathrm{VP}} \quad \mathrm{t}$ ]

This time, it is the Humanness Exclusion Principle that eliminates the derivation in (a), which violates that principle, in favor of the derivation in (b), which obeys that principle. The Specificity Exclusion Principle is not violated by either derivation (because the object is not singular), so it has no effect here.

Let us now consider an example involving a non-human object that is singular, but non-specific. The example below is ambiguous between non-specific singular, non-specific plural, and specific plural, but because we need to compare derivations with fixed features on the objects (see section four), let us focus in on the derivation with a singular, non-specific object:

(50) Te-'illebed a bilis a rengalek. 3p-Pf-hit dog children

The kids hit a $\operatorname{dog} /$ the $\operatorname{dogs} /$ some $\operatorname{dog}(\mathrm{s})$. (Georgopoulos 1991, p. 30)
a.
[VP $\operatorname{dog}[$-specific, +singular] ]
b. $\operatorname{dog}[-$ specific, +singular $]$ Agr-O [vP $\quad$ t $]$

Neither derivation violates the Humanness Exclusion Principle, so that has no effect here. Because the object is non-specific, neither derivation violates the Specificity Exclusion Principle, so that also has no effect here. Thus, in this situation, the economy principle, Avoid Movement, gets to make the decision, and it eliminates the (b) derivation, because it involves one violation of Avoid Movement, while the (a) derivation involves no violations. Thus we get the correct result that the object in this construction will remain in its base position and not trigger object agreement: 
(52) (Object is [+singular, -specific, -human])

\begin{tabular}{|c|c|c|c|}
\hline \multicolumn{2}{|c|}{ Candidates } & Exclusion & Avoid \\
\hline a. & $\begin{array}{l}\text { Object in } \\
\text { Base Position }\end{array}$ & & \\
\hline b. & $\begin{array}{l}\text { Object Moves } \\
\text { to Spec Agr-O }\end{array}$ & & $* !$ \\
\hline
\end{tabular}

The effect of the operation of these principles is the desired one, that objects will not trigger object agreement unless they are [+human] and/or [+singular and +specific].

Now, let us turn to double object constructions. Let us address the question of what happens if both objects have features that would violate the exclusion principles if these objects remain in the VP. In the following example, the goal object is human and the theme object can be interpreted as singular and specific. The first object must trigger object agreement, but the second object cannot trigger object agreement, regardless of its interpretation.

(53) Ak-mils-terir a resechelik a hong.

$1 \mathrm{~s}-$ gave-3p friends book

I gave my friends a/the book. (Josephs 1975, p. 347)

Let us focus on the interpretation in which the theme object, book, is specific and singular. We need to compare the following set of candidates, where neither object moves (a), or the first object moves (b), or the second object moves (c): $:^{10}$

a.

[VP friends the-book ]

b. friends Agr-O [ ${ }_{\mathrm{VP}} \quad \mathrm{t}$ the-book $]$

c. the-book Agr-O [VP friends $\mathrm{t} \quad]$

Here, all of the candidates violate at least one of the Exclusion Principles. The candidate in (a) violates both the Humanness and the Specificity Exclusion Principles. The candidate in (b) violates the Specificity Exclusion Principle, because 'the-book' remains in the VP, while the candidate in (c) violates the Humanness Exclusion Principle, because 'friends' remains in the VP. The

\footnotetext{
${ }^{10}$ It is assumed here that the derivation in which both objects move out of the VP is impossible because there is only one Spec Agr-O position available and movement to any other position would not be movement to a Cased position (thus ruled out by the Case Filter).
} 
derivation that would obey both exclusion principles (where both objects move) is ruled out for independent reasons, as noted the previous footnote.

Since we know that the (b) derivation is the one selected, one might think that this example provides an argument for ranking the exclusion principles with respect to each other, with the Humanness Exclusion Principle ranked higher, so that when conditions are such that both principles cannot be satisfied, it is the human object that must move. However, the descriptions in Josephs 1975 and Georgopoulos 1991, 1992 make it clear that it is always the first object that triggers agreement, and never the second, and no qualification is mentioned regarding the features of those objects. Thus, although no example is given with two human or two non-human objects, the implication is that the only the first object would trigger agreement in these sorts of examples as well.

Thus, in the absence of any clear evidence for any internal ranking of the exclusion principles, they will be considered here as a group. The derivation in (a) above violates the exclusion principles twice, while the derivations in (b) and (c) violate these principles only once. Thus the exclusion principles eliminate (a) because there are better derivations available in (b) and (c). The exclusion principles do not eliminate either (b) or (c) because there are is no better derivation available. With respect to the Avoid Movement, (b) and (c) are also equivalent because each involves one movement.

(55) First object [+human]; second object [+singular, +specific, -human]

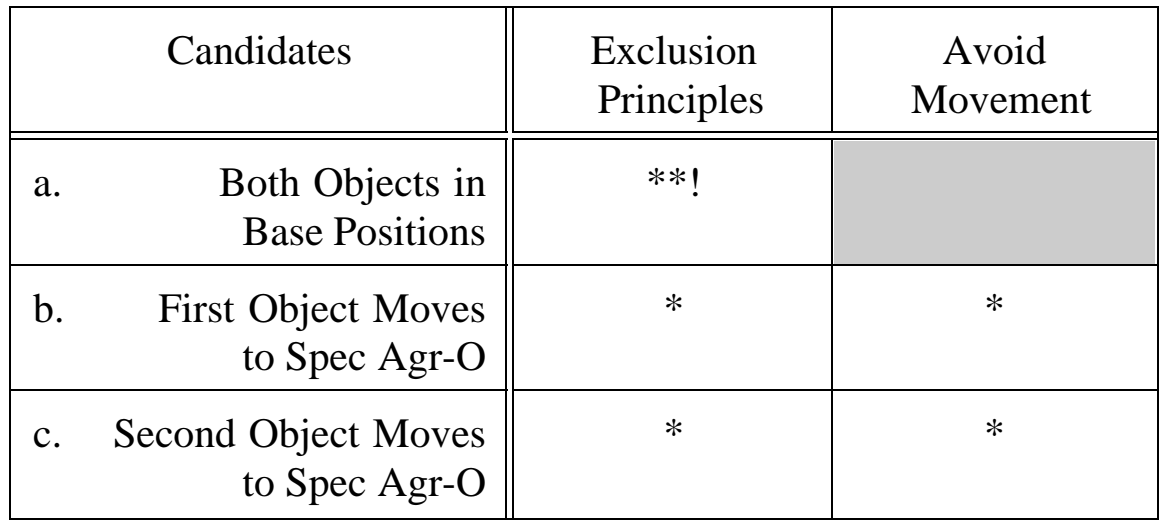

However, since Palauan does not allow the derivation in (c) to surface, something else must be ruling out (c). I will assume for now that some version of minimality prevents the second object from moving over the first object, ruling out (c). ${ }^{11}$

\footnotetext{
${ }^{11}$ Whatever the principle is that prevents the second object from moving over the first object, this principle is violable in some languages (at least when the alternative is a violation of the Case Filter), since the second object can move over the first in languages with a symmetric passive pattern (see Woolford 1993).
} 
Thus, in this example, the best derivation available still involves one violation of the exclusion principles. Because this derivation is grammatical, it shows that the exclusion principles are violable under the right circumstances.

To summarize this section, we have seen that the economy principles prevent movement to Spec Agr-O, unless that movement is necessary to prevent a violation of one or more of the exclusion principles. However, we have also seen that when movement to Spec Agr-O is not possible (because the node is filled), the exclusion principles can be violated.

Let us now turn to imperfective constructions.

\subsection{Imperfective Constructions}

The same objects that trigger object agreement in perfective constructions get marked by preposition in imperfective constructions, as we saw in section one. Thus P Insertion appears to be an alternate strategy for avoiding violations of the Exclusion Principles.

In this section, we will first deal with the question of exactly how $P$ Insertion changes a derivation so that it no longer violates the exclusion principles. Following this, the details are presented of how the interaction of exclusion principles and the economy principle, Avoid Insertion, produces the range of data in imperfective clauses discussed in section one.

\subsubsection{What P Insertion Does}

There are two possible answers to the question of how $\mathrm{P}$ Insertion avoids violations of the exclusion principles. Either one of these answers would be compatible with the analysis being developed here. One possibility is that NPs inside PPs are subject to the exclusion principles, just as bare objects are, but that the creation of a PP makes movement out of the VP possible (because it is easier to move a PP than an NP). To be consistent, one would also have to assume that all PPs containing specific NPs move out of the VP. The other possibility, which will be assumed here, is that NPs inside PPs are exempt from the exclusion principles. That is, that the exclusion principles only refer to the features of objects governed by $\mathrm{V}$. Under that view, the function of $\mathrm{P}$ insertion is to block government by $\mathrm{V}$, making an NP exempt from the requirements of exclusion principles. Let us reformulate the exclusion principles in this manner: ${ }^{12}$

\footnotetext{
${ }^{12}$ Diesing and Jelinek (1994) consider the question of whether or not the Mapping Hypothesis applies to PPs to be an open one.
} 
a. Specificity Exclusion Principle (Palauan Variant)

NPs inside the VP, governed by V cannot be [+specific, +singular].

b. Humanness Exclusion Principle

NPs inside the VP, governed by V cannot be [+human].

\subsubsection{Imperfect Clauses}

The behavior of imperfect clauses follows from the interaction of the exclusion principles and the second economy principle, Avoid Insertion. Let us begin with an imperfective clause with a non-human object that is specific and singular. We see that this example has P Insertion:

a. Ng-milengelebed er a bilis.

3s-Im-hit $\quad P \quad \operatorname{dog}$

S/he hit the dog.

The two candidates the grammar needs to compare are the derivation in which the object remains unmarked in its base position and the derivation in which there is $\mathrm{P}$ Insertion. (Sections six and seven will answer the question of what blocks movement to Spec Agr-O in imperfect constructions.)

a. [VP $\quad \operatorname{dog}[+$ specific,, singular $] \quad]$

b.

$$
\left[_{\mathrm{VP}} \quad\left[\mathrm{PP}_{\mathrm{P}} \mathrm{P} \operatorname{dog}[+ \text { specific, }+ \text { singular }]\right] \quad\right]
$$

As in the perfective examples, we would get the wrong result if we allowed the economy principle, Avoid Insertion, to operate first, eliminating the derivation with P Insertion. Instead, the exclusion principles must apply first. The Specificity Exclusion Principle is violated by the derivation in (a), where a singular specific object occupies a position inside VP that is governed by the verb. ${ }^{13}$ This exclusion principle is not violated by the (b) derivation where $\mathrm{P}$ Insertion blocks government by the verb. Thus the (a) derivation is eliminated in favor of (b):

\footnotetext{
${ }^{13}$ Since the verb may have moved, we need to consider government by the trace of a verb to have the same effect under the exclusion principles as government by the verb itself.
} 
Exclusion Principles $\gg$ Avoid Insertion

(Object is [+singular, +specific, -human])

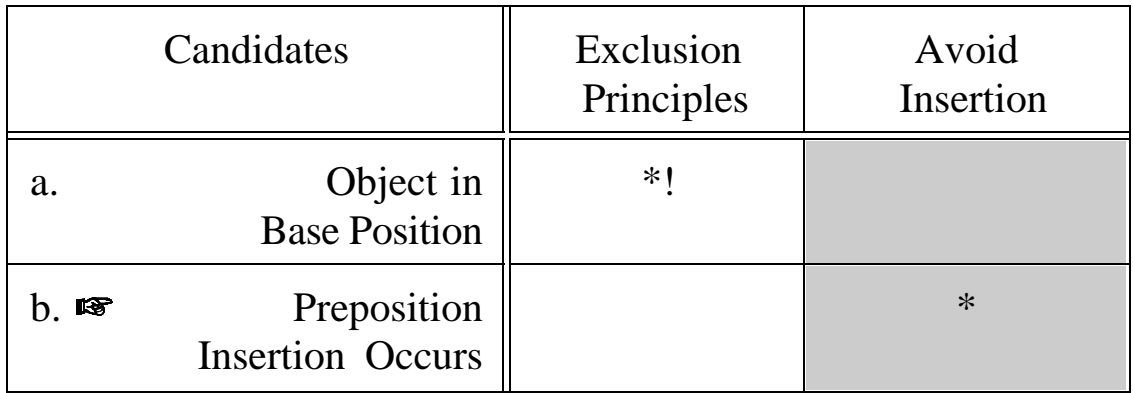

Although the (b) derivation violates Avoid Insertion, this is irrelevant because the selection of the best candidate has already been made before Avoid Insertion has a chance to apply.

In an example with a non-specific, non-human object, there is no P Insertion; thus, we want the (a) derivation to be selected as the best derivation:

(60) Ak ousbech a bilas er a klukuk.

I need boat tomorrow

I need a boat tomorrow. (Josephs 1975 p. 49)

We compare the following two candidates, without and with P Insertion:
a.
$[\mathrm{VP}$
boat [-specific, + singular] ]
b.
$\left[{ }_{\mathrm{VP}} \quad\left[{ }_{\mathrm{PP}} \mathrm{P}\right.\right.$ boat $[-$ specific, + singular $\left.\left.]\right] \quad\right]$

Here candidate (a) does not violate either exclusion principle, because the object is not human, nor is it both specific and singular. Since both candidates obey the exclusion principles, both candidates are passed on to the economy principles for a final selection of the best candidate. Now the economy principle, Avoid Insertion, eliminates the (b) candidate in favor of the (a) candidate:

(62) (Object is [+singular, -specific, -human])

\begin{tabular}{|c|c|c|c|}
\hline \multicolumn{2}{|c|}{ Candidates } & Exclusion & Avoid \\
\hline a. & $\begin{array}{r}\text { Object in } \\
\text { Base Position }\end{array}$ & & \\
\hline b. & $\begin{array}{l}\text { Preposition } \\
\text { sertion Occurs }\end{array}$ & & $* !$ \\
\hline
\end{tabular}

The treatment of double object constructions is more straightforward in imperfective constructions than in perfectives. Since both objects can undergo P 
Insertion if they would otherwise violate the exclusion principles, each object is treated just like a single object in the examples above. In the example below, the first object is human; thus, the Humanness Exclusion Principle would be violated if this object were not marked by $\mathrm{P}$ Insertion. The second object is both singular and specific; thus, the Specificity Exclusion Principle would be violated if it were not embedded in a PP.

A Romana a omeka er a rengalek er a kukau.

Romana feed $\mathrm{P}$ children $\mathrm{P}$ taro

Romana is feeding the children the taro. (Josephs 1975, p. 207)

\subsection{P Insertion in Other Languages}

Palauan is not the only language to use $\mathrm{P}$ Insertion to avoid violating exclusion principles. This also occurs in Spanish. Spanish shows evidence of the operation of an exclusion principle involving specificity and humanness. Inanimate objects are unmarked, while specific human objects are marked with the preposition a (Comrie 1981). ${ }^{14}$ Spanish is like imperfective constructions in Palauan, in that only P Insertion, but not movement to Spec Agr-O with object agreement, is used to avoid violating the exclusion principles.

Not all languages make use of $\mathrm{P}$ Insertion to avoid violations of exclusion principles. Swahili behaves like perfective constructions in Palauan. Human objects and nonhuman definite/specific objects obligatorily trigger object agreement (Givon 1976) and P Insertion is not an option. 15

The fact that this difference in perfective and imperfective aspects in Palauan (the use of movement to Spec Agr-O versus P Insertion) is mirrored in cross-linguistic differences between languages like Spanish and Swahili will be an important factor motivating the treatment of this aspectual difference in Palauan developed in the next section.

\footnotetext{
${ }^{14}$ The question of exactly when objects are marked with a in Spanish is controversial and may be complicated by the interaction of other factors. Thus no claim is being made here about the exact nature (or number) of exclusion principles operating in Spanish.

${ }^{15}$ Givon 1976 mentions a number of other languages in which the dative preposition is used to mark definite objects. It may be possible to analyze such languages along the lines suggested here for imperfective constructions in Palauan. By making use of a small family of exclusion principles, based on features such as [+specific], [+singular], [+human], and perhaps [+animate], we may be able to account for many of the phenomena discussed in the typological literature under the heading of animacy hierarchies (see Comrie 1981).
} 


\section{The Problem of Aspect}

The analyses presented above for perfective and imperfective constructions treat object marking in aspect in isolation. While both analyses require the same exclusion principles, they make use of different economy principles. Nothing has yet been said about the role of the other economy principle. The problem now is to integrate these analyses, preferably in a way that answers the question of how aspect determines whether movement or preposition insertion will be used to avoid violations of the exclusion principles in Palauan.

The first part of this section will show that, using current conventional syntactic mechanisms, only a redundant and stipulative account of the role of aspect is possible. The second part of this section shows that a very simple and complete account is possible if we make use of the mechanisms available in Optimality Theory to a greater extent than we have so far; that is, if we make use of re-ranking. We will see that all of the facts described in section one fall out directly if the rank of one of the economy principles changes in the perfective aspect.

\subsection{A Conventional Approach to the Effect of Aspect}

It is fairly easy to prevent movement to Spec Agr-O (and thus object agreement) in imperfective constructions in Palauan using conventional syntactic means. We could propose that Aspect provides the objective Case feature assigned by Agr-O, just as Tense supplies the nominative Case feature that is assigned/checked by Agr-S (see, for example Chomsky 1992). This would be a change from the assumption in Chomsky 1992 that it is the verb that raises to Agr-O to supply this feature, but the parallelism with nominative Case is attractive. ${ }^{16}$

We could then account for the fact that movement to Spec Agr-O only occurs in the perfective aspect in Palauan by stipulating that the Aspect node only has a Case feature to supply if it is [+perfective]. In a language like Spanish, where it appears that Agr-O is never available, one could stipulate that no Aspect has a Case feature to supply. In contrast, one would claim that all aspects have a Case feature in a language like Swahili.

While this provides a nice account of the absence of movement to Spec Agr-O in imperfective clauses in Palauan, we are left with the much more difficult question of why $\mathrm{P}$ Insertion is not available in perfective clauses.

Recall that $\mathrm{P}$ Insertion is not available in perfectives, even when movement to Spec Agr-O is not an option, i.e. for the second object in a double object

\footnotetext{
${ }^{16}$ Independent evidence that $\mathrm{V}$ does not supply the Case feature assigned by Agr-O is presented in Woolford 1994.
} 
construction. Thus, no solution that merely makes movement the preferred option over P Insertion will account for all of the facts. If we stipulate that there is some feature associated with aspect that determines whether or not P Insertion can be used, call it [+/- P Insertion available], we can make the analysis work, but we have a conceptual problem above and beyond the stipulative nature of such an approach.

The conceptual problem with such an approach is that it is redundant. It has two ways of blocking movement and two ways of blocking insertion. One way is by means of economy principles and the other involves features on the aspect node:

Two Ways to Block Movement

a. Economy Principle: Avoid Movement

b. Aspect node lacks a Case feature

Two Ways to Block P Insertion

a. Economy Principle: Avoid Insertion

b. Aspect node lacks some feature

It is possible to eliminate this redundancy and make the economy principles do all of this work if we rank the principles correctly. This is the goal of the next section.

\subsection{An Optimality Solution}

We saw at the end of section five that the kind of difference we find between the two aspects within Palauan is the kind of cross-linguistic difference that characterizes Spanish versus Swahili. Like imperfective constructions in Palauan, Spanish only allows P Insertion, not movement to Spec Agr-O, to avoid violations of the exclusion principles. Like perfective constructions in Palauan, Swahili only allows the movement option, and not P Insertion. It is proposed here that the difference in the two aspects of Palauan should be treated just as Optimality Theory would treat such a difference in two distinct languages such as Spanish and Swahili; that is, by ranking the principles differently. ${ }^{17}$

The idea is that all syntactic devices such as $\mathrm{P}$ Insertion and Movement to Spec Agr-O are theoretically available in every construction in every language.

\footnotetext{
${ }^{17}$ The idea of partial re-ranking of principles within one language has been proposed in work on phonology in the OT framework in work such as Kisseberth 1993, Rosenthall 1994, Cohn and McCarthy 1994, and Itô and Mester 1995.

Some of the questions that arise when re-ranking is proposed in syntax will be dealt with at the end of this section.
} 
However, these devices are 'expensive' and avoided if possible. Languages differ as to how 'expensive' each device is and this difference is expressed in the ranking of the economy principles with respect to other principles.

Let us first consider the ranking of principles that accounts for the facts of imperfective constructions in Palauan.

\subsubsection{Ranking in Imperfective Constructions}

We saw in section five that Avoid Insertion has to be ranked below the exclusion principles in order to produce the correct results:

\section{Exclusion Principles 》 Avoid Insertion}

The question now is where does the other economy principle, Avoid Movement, belong in this ranking. Since imperfect constructions behave as if movement is costlier than insertion, we should rank Avoid Movement above Avoid Insertion to express this generalization.

\section{Avoid Movement $\gg$ Avoid Insertion}

This ranking essentially says that it is more important to Avoid Movement than it is to Avoid Insertion. Thus, whenever a situation arises in which either movement or insertion would prevent a violation of a higher ranked principle, one will chose to use P Insertion rather than movement. However, movement could still be chosen over insertion if only movement would satisfy a higher ranked principle. Thus, this ranking will not prevent subjects from moving in order to satisfy the Extended Projection Principle, since P Insertion will not do the job in that situation.

What we do not know from the facts available in imperfective constructions in Palauan, is how (or even whether) to rank the exclusion principles with respect to Avoid Movement. We could determine this if there were ever a situation in which $\mathrm{P}$ Insertion were not available to prevent a violation of the exclusion principles. Then we could tell if it is more important to obey the exclusion principles or to avoid movement. However, $\mathrm{P}$ Insertion is always possible in Palauan imperfectives, even for both objects in double object constructions. The only evidence we have of the ranking of these principles is their behavior in perfective clauses, where the exclusion principles are ranked higher. Assuming that a child acquiring Palauan will assume that rankings in the two aspects are alike, unless he is presented with evidence to the contrary, we can tentatively assume the following ranking for imperfective constructions in Palauan.

(68) Palauan Ranking in the Imperfective Aspect:

Exclusion Principles $\gg$ Avoid Movement $\gg$ Avoid Insertion 
Given this ranking, the facts of imperfective clauses fall out just as described above in section five, without the necessity of any additional stipulation to block movement. Example tableaus demonstrating how this ranking selects among derivations with and without movement and insertion, given objects with various combinations of features, will be given in section seven. Before we get into these technical details, let us turn to the ranking that is necessary to get the facts in the perfective aspect to fall out neatly.

\subsubsection{Ranking in Perfective Constructions}

We saw in section five that it is necessary for Avoid Movement to be ranked lower than the exclusion principles:

\section{Exclusion Principles 》 Avoid Movement}

The fact that $\mathrm{P}$ Insertion is never used in perfective constructions, not even to prevent a violation of the exclusion principles, suggests that Insertion is extremely expensive here, too expensive to use in any of the constructions discussed here. We can express this degree of costliness by ranking Avoid Insertion higher than either of the other principles:

(70) Palauan Ranking in the Perfective Aspect:

\section{Avoid Insertion $\gg$ Exclusion Principles $\gg$ Avoid Movement}

This ranking will account for the perfective data exactly as described in section five and it solves the problem of ruling out all derivations with $\mathrm{P}$ Insertion. Example tableaus will be given below in section seven, but first let us consider a question that the use of re-ranking in syntax raises.

\subsection{Where is ranking information stored?}

One question that arises when the idea of re-ranking is proposed for syntax is where is information about principle ranking stored. There is no reason to think that the answer to this question is any different than the answer to the standard question of where information about parametric differences between languages is stored. One fairly standard idea is that such differences are located in the lexicon. For Palauan, the ranking information discussed in this paper must be coded on the Aspect node or the Aspect morpheme. However, it is not necessary for all ranking information to be coded here. Instead, it is possible that bits of ranking information, perhaps involving only the rankings between a few principles, might be located on different lexical items and that no total ranking of all principles would be specified anywhere, but rather the total ranking would only be constructed or deduced by combining the partial rankings. 
In the work on language internal re-ranking in phonology cited above, generally only one principle changes its ranking and that is true here as well. If the ranking in the imperfect is the default ranking for the language, then the only information that has to be coded in the perfective is that the ranking of one principle, Avoid Insertion, changes from lowest to highest.

In any approach to the Palauan data, it would be necessary to claim that there is some sort of language particular information coded on the Aspect node or morpheme because the properties of perfective and imperfective aspect constructions are different. One advantage of coding this aspect specific information in the form of ranking information, instead of some more conventional syntactic features on aspect, is that re-ranking provides a means of accounting for non-local effects of aspect in the clause, such as P Insertion on objects in Palauan. A similar approach might account for the fact that aspect determines the choice between Case patterns (nominative-accusative vs. ergative-absolutive) in many split ergative languages such as Hindi (e.g. Mahajan 1990).

\section{Summary of The Optimality Solution for Palauan Object Marking}

The principles used in this account (constraints, in OT terminology) to select the derivation/representation that surfaces in Palauan are summarized below. For Palauan, we need two economy principles and two exclusion principles:

(71) Economy Principles:

a. Avoid Movement

b. Avoid Insertion

$$
\text { Exclusion Principles: }{ }^{18}
$$

a. Specificity Exclusion Principle:

NPs inside VP, governed by V, cannot be [+specific, +singular].

b. Humanness Exclusion Principle:

NPs inside VP, governed by V cannot be [+human].

\footnotetext{
${ }^{18}$ It is proposed that there is a family of Exclusion Principles available in UG which refer to features such as [+specific], [+singular], [+human], and [+animate]. The idea of a family of related principles replaces (or expresses) the idea suggested in Comrie 1981 and Diesing and Jelinek 1993 that languages can differ in what counts as specific.
} 
With respect to the ranking of these constraints, the two exclusion principles form an internally unranked group, ranked with respect to the other principles as follows:

\section{Exclusion Principles 》Avoid Movement 》 Avoid Insertion}

The perfect aspect node or morpheme induces a partial re-ranking of these principles: the economy principle that is ranked lowest in the imperfective is elevated to the highest rank among these principles in the perfective.

Ranking in the Perfective Aspect:

\section{Avoid Insertion 》 Exclusion Principles 》 Avoid Movement}

In this account, the Candidate Set (the set of structures that are compared to determine the best candidate) is defined essentially as in Grimshaw 1994 and Chomsky 1994. The structures to be compared are all those that can be derived from a common Input (a fixed set of lexical items with fixed features and the structure(s) that can be projected from these lexical items), with variant candidates being produced by the application (or not) of NP Movement or P Insertion.

It is assumed that verbs assign Case only optionally in Palauan (if not universally). Thus for any construction containing an object, there are at least three derivations that are consistent with the Case Filter. When the verb assigns case to its object, the only derivation that does not violate the Case Filter is the one in which the object remains in its base position. When the verb does not assign Case to its object, two possible derivations are produced that obey the Case Filter. In one, the object moves to Spec Agr-O for Case and in the other the object is assigned Case by preposition insertion. 19

\subsection{Example Tableaus: Single Object Constructions}

For all of the single object constructions exemplified below, three candidate derivations are compared:

a. The object remains in its base position:

$$
\left[\begin{array}{lll}
\mathrm{VP} & \mathrm{NP}
\end{array}\right.
$$

b. The object moves to Spec Agr-O, triggering object agreement:

$$
\mathrm{NP}_{\mathrm{i}} \quad \text { Agr-O } \quad\left[\begin{array}{lll}
\mathrm{VP} & \left.\mathrm{t}_{\mathrm{i}} \quad\right]
\end{array}\right.
$$

\footnotetext{
${ }^{19}$ Although the derivations that violate the Case Filter are technically members of the Candidate set as well, they will be ignored here.
} 
c. The object is marked by preposition insertion:

$$
\left[\begin{array}{llll}
\mathrm{VP} & {\left[\begin{array}{lll}
\mathrm{PP} & \mathrm{P} & \mathrm{NP}
\end{array}\right]}
\end{array}\right]
$$

\subsubsection{Perfective Aspect}

Let us begin with a construction in which the object has features that would violate both exclusion principles if it remained in its base position. This is the situation when the object is both [+human] and [+specific, +singular]. Since Avoid Insertion is the highest ranked principle, the candidate involving $\mathrm{P}$ Insertion, (c), is eliminated first. The exclusion principles then eliminate the derivation in (a) in which the object remains in its base position. This leaves only one remaining candidate, (b). Thus (b) is the best candidate, even though it violates the lowest ranking principle, Avoid Movement:

(76) Object is [+human] and [+specific, + +singular $]$

\begin{tabular}{|c|c|c|c|c|}
\hline \multicolumn{2}{|r|}{ Candidates } & $\begin{array}{l}\text { Avoid } \\
\text { Insertion }\end{array}$ & $\begin{array}{l}\text { Exclusion } \\
\text { Principles }\end{array}$ & $\begin{array}{c}\text { Avoid } \\
\text { Movement }\end{array}$ \\
\hline a. & $\begin{array}{c}\text { Object in } \\
\text { Base Position }\end{array}$ & & $* ! *$ & \\
\hline b. & $\begin{array}{l}\text { Object Moves } \\
\text { to Spec Agr-O }\end{array}$ & & & $*$ \\
\hline c. & Insert P & $* !$ & & \\
\hline
\end{tabular}

The result is essentially the same if the object has features that only violate one of the two exclusion principles:

(77) Object is [+human] and either [-specific] or [-singular] or else [-human] and [+specific, +singular]

\begin{tabular}{|lr||c|c|c|}
\hline & Candidates & $\begin{array}{c}\text { Avoid } \\
\text { Insertion }\end{array}$ & $\begin{array}{c}\text { Exclusion } \\
\text { Principles }\end{array}$ & $\begin{array}{c}\text { Avoid } \\
\text { Movement }\end{array}$ \\
\hline \hline a. & $\begin{array}{r}\text { Object in } \\
\text { Base Position }\end{array}$ & & $* !$ & \\
\cline { 1 - 4 } b. & $\begin{array}{r}\text { Object Moves } \\
\text { to Spec Agr-O }\end{array}$ & & & $*$ \\
\cline { 1 - 4 } c. & Insert P & $* !$ & & \\
\hline
\end{tabular}

If the object violates neither exclusion principle when it remains in its base position, then the situation changes because that derivation, (a), does not violate 
any of the principles. As above, the $\mathrm{P}$ Insertion derivation is eliminated immediately, but now both remaining derivations obey the exclusion principles. Thus the decision between the two remaining derivations is made by the lowest ranking principle, Avoid Movement, which eliminates the derivation involving movement to Spec Agr-O, (b). Thus (a) is the derivation that surfaces:

(78) Object is [-human] and ([-specific] and/or [-singular])

\begin{tabular}{|c|c|c|c|c|}
\hline \multicolumn{2}{|r|}{ Candidates } & $\begin{array}{l}\text { Avoid } \\
\text { Insertion }\end{array}$ & $\begin{array}{l}\text { Exclusion } \\
\text { Principles }\end{array}$ & $\begin{array}{c}\text { Avoid } \\
\text { Movement }\end{array}$ \\
\hline a. & $\begin{array}{r}\text { Object in } \\
\text { Base Position }\end{array}$ & & & \\
\hline b. & $\begin{array}{l}\text { Object Moves } \\
\text { to Spec Agr-O }\end{array}$ & & & $* !$ \\
\hline c. & Insert $\mathrm{P}$ & $* !$ & & \\
\hline
\end{tabular}

\subsubsection{Imperfective Aspect}

Let us now consider the same situations in the imperfective aspect, where the ranking of the principles is slightly different. Again, let us begin with an example in which the object has features referred to by one or both exclusion rules: [+human] and/or [+specific, +singular]. Since the exclusion principles are now ranked highest, they eliminate the base generated derivation in (a). (If the object is both human and specific and singular, this will result in two violations.) The next highest principle, Avoid Movement, then eliminates the derivation involving movement to Spec Agr-O, (b), leaving the derivation in (c), involving P Insertion, as the only remaining, and therefore best derivation of the three. The fact that (c) violates the lowest ranked principle, Avoid Insertion, is irrelevant.

(79) Object is [+human] and/or [+specific, +singular]

\begin{tabular}{|c|c|c|c|c|}
\hline \multicolumn{2}{|c|}{ Candidates } & $\begin{array}{l}\text { Exclusion } \\
\text { Principles }\end{array}$ & $\begin{array}{c}\text { Avoid } \\
\text { Movement }\end{array}$ & $\begin{array}{l}\text { Avoid } \\
\text { Insertion }\end{array}$ \\
\hline a. & $\begin{array}{l}\text { Object in } \\
\text { Base Position }\end{array}$ & $* !(*)$ & & \\
\hline b. & $\begin{array}{l}\text { Object Moves } \\
\text { to Spec Agr-O }\end{array}$ & & $*$ & \\
\hline c. & Insert $\mathrm{P}$ & & & $*$ \\
\hline
\end{tabular}


On the other hand, if the object has features that would not violate either exclusion principle, then the best derivation is the one in which the object remains in its base position, because that derivation, (a), does not violates any of these principles:

(80) Object is [-human] and either [-specific] and/or [-singular]

\begin{tabular}{|c|c|c|c|}
\hline Candidates & $\begin{array}{l}\text { Exclusion } \\
\text { Principles }\end{array}$ & $\begin{array}{c}\text { Avoid } \\
\text { Movement }\end{array}$ & $\begin{array}{l}\text { Avoid } \\
\text { Insertion }\end{array}$ \\
\hline $\begin{array}{r}\text { Object in } \\
\text { a. } \\
\text { Base Position }\end{array}$ & & & \\
\hline $\begin{array}{l}\text { Object Moves } \\
\text { to Spec Agr-O }\end{array}$ & & $* !$ & \\
\hline Insert $\mathrm{P}$ & & & $* !$ \\
\hline
\end{tabular}

This exhausts the feature possibilities for a single object. It happens that the best derivation in single object constructions always obeys the exclusion principles. This does not always hold in double object constructions, to which we now turn. Here, we see that the exclusion principles are violable.

\subsection{Double Object Constructions}

In the examples of double object constructions to be discussed below, we will focus on the behavior of the second object because the first object behaves like a single object in the above examples. In all of the candidates to be compared below, the first object will be held constant at [+human], and assumed to move to Spec Agr-O in perfectives, or to be marked with $\mathrm{P}$ Insertion in imperfectives. Thus we will only be comparing derivations with variation in the features and position of the second object:

a. The second object remains in its base position.

b. The second object moves to Spec Agr-O, triggering object agreement.

c. The second object is marked by preposition insertion:

\subsubsection{Double Objects in the Perfective Aspect}

In the perfective aspect, the (b) option will either not be generated (because there is only one Spec Agr-O and it is filled with the first object) or else movement of the second object to that position will violate minimality and 
the derivation will be eliminated. Thus we will compare only the (a) and (c) derivations.

Let us first consider the situation involving a second object with features that do not violate any of the exclusion principles. Here, the derivation in (c) with P Insertion is eliminated by the highest ranking constraint, leaving the base generated derivation in (a) as the best derivation.

\begin{tabular}{|c|c|c|c|c|}
\hline \multicolumn{2}{|r|}{ Candidates } & $\begin{array}{l}\text { Avoid } \\
\text { Insertion }\end{array}$ & $\begin{array}{l}\text { Exclusion } \\
\text { Principles }\end{array}$ & $\begin{array}{c}\text { Avoid } \\
\text { Movement }\end{array}$ \\
\hline a. & $\begin{array}{l}\text { 2nd Object in } \\
\text { Base Position }\end{array}$ & & & \\
\hline c. 1 & Insert P (2nd obj.) & $* !$ & & \\
\hline
\end{tabular}

The same candidate wins even if the second object has features that violate one or both of the exclusion principles. The P Insertion derivation, (c) is still eliminated first, leaving the base generated (a) derivation as the best derivation available, even though it violates one or both exclusion principles.

(83) Second Object is [+human] and/or [+specific and +singular]

\begin{tabular}{|l||c|c|c|}
\hline Candidates & $\begin{array}{c}\text { Avoid } \\
\text { Insertion }\end{array}$ & $\begin{array}{c}\text { Exclusion } \\
\text { Principles }\end{array}$ & $\begin{array}{c}\text { Avoid } \\
\text { Movement }\end{array}$ \\
\hline \hline a. $\begin{array}{r}\text { 2nd Object in } \\
\text { Base Position }\end{array}$ & & $*(*)$ & \\
\hline c. Insert P (2nd obj.) & $* !$ & & \\
\hline
\end{tabular}

Because insertion is so expensive and movement is not available to the second object, there is no way for the second object in a perfective construction to obey the exclusion principles in Palauan.

\subsubsection{Double Objects in the Imperfective Aspect}

Things work out somewhat differently in the imperfective aspect because there is no limit on how many objects can undergo P Insertion at the same time, unlike the limit of one object moving to Spec Agr-O.

Let us consider the situation in which the second object has features that would violate one or both of the exclusion principles. The base generated (a) derivation is eliminated first, due to one or more violations of the exclusion 
principles. This leaves (b) and (c). The (b) derivation is elimiminated next, by Avoid Movement. ${ }^{20}$ This leaves the P Insertion derivation in (c) as the best derivation, even though it violates Avoid Insertion:

(84) Second Object is [+human] and/or [+specific and +singular]

\begin{tabular}{|c|c|c|c|}
\hline Candidates & $\begin{array}{c}\text { Exclusion } \\
\text { Principles }\end{array}$ & $\begin{array}{c}\text { Avoid } \\
\text { Movement }\end{array}$ & $\begin{array}{l}\text { Avoid } \\
\text { Insertion }\end{array}$ \\
\hline $\begin{array}{l}\text { 2nd Object in } \\
\text { Base Position }\end{array}$ & $* !(*)$ & & \\
\hline $\begin{array}{r}\text { b. 2nd Object Moves } \\
\text { to Spec Agr-O }\end{array}$ & & $* !$ & \\
\hline c. Insert P (2nd obj.) & & & * \\
\hline
\end{tabular}

If the features of the second object are such that there is no violation of any of the exclusion principles in the (a) derivation, then (a) emerges as the best derivation because it does not violate any of the principles, while (b) is eliminated by Avoid Movement and (c) by Avoid Insertion.

(85) Second Object is [-human] and either [-specific] and/or [-singular]

\begin{tabular}{|c|c|c|c|}
\hline Candidates & $\begin{array}{l}\text { Exclusion } \\
\text { Principles }\end{array}$ & $\begin{array}{c}\text { Avoid } \\
\text { Movement }\end{array}$ & $\begin{array}{l}\text { Avoid } \\
\text { Insertion }\end{array}$ \\
\hline $\begin{array}{ll}\text { a. } & \text { 2nd Object in } \\
& \text { Base Position }\end{array}$ & & & \\
\hline $\begin{array}{r}\text { b. 2nd Object Moves } \\
\text { to Spec Agr-O }\end{array}$ & & *! & \\
\hline c. Insert P (2nd obj.) & & & $* !$ \\
\hline
\end{tabular}

${ }^{20}$ Recall that the (b) derivation also violates minimality and is thus technically already eliminated at this point. 


\section{Conclusions}

This paper has shown that what initially appears to be a complex, language specific pattern of object agreement and preposition insertion in Palauan follows from the interaction of a small number of principles, none of which are unique to Palauan. Two economy principles, Avoid Movement and Avoid Insertion, work to keep objects in their base positions. At odds with these economy principles are mapping/exclusion principles that exclude objects with certain features from object positions inside the VP. Palauan manifests mapping/exclusion principles based on specificity (as in Diesing 1992) and on humanness.

There are two ways to avoid violating mapping/exclusion principles. One is to move the object out of the VP, to Spec Agr-O for example, as suggested in Diesing 1992. This strategy is used in perfective clauses in Palauan and in languages like Swahili. The other strategy is to insert a preposition. The presence of a preposition blocks government by the verb and makes an object exempt from exclusion principles. The preposition insertion strategy is used in imperfectives in Palauan and in languages like Spanish. Each of these strategies violates one of the economy principles and what determines which strategy will be chosen is the relative ranking of these economy principles (expressing the notion of whether movement or insertion is considered more costly).

The striking difference that aspect makes in the behavior of objects in Palauan cannot be easily accounted for using conventional syntactic mechanisms, but it follows directly if the rank of one of the economy principles, Avoid Insertion, changes in the perfective aspect. Treating the effect of aspect on the syntax of clauses in terms of principle re-ranking offers a means of accounting for the non-local effects that the aspect node can have on the clause, such as determining whether or not preposition insertion is available to objects in Palauan or determining whether or not the subject can have ergative Case in split ergative languages such as Hindi. 


\section{References}

Burzio, Luigi. 1986. Italian syntax: A government-binding approach. Dordrecht: Reidel.

Chomsky, Noam. 1981. Lectures on government and binding. Dordrecht: Foris.

Chomsky, Noam. 1989. Economy of derivations and representations. In I. Laka and Mahajan eds. MITWPL 10:43-74.

Chomsky, Noam. 1992. A minimalist program for linguistic theory. MIT Occasional Papers in Linguistics 1.

Chomsky, Noam. 1994. Bare phrase structure. MIT Occasional Papers in Linguistics 5.

Cohn, Abigail and John McCarthy. 1994. Alignment and parallelism in Indonesian phonology. Ms., Cornell University and University of Massachusetts, Amherst.

Comrie, Bernard. 1981. Language universals and linguistic typology. Oxford: Basil Blackwell.

Diesing, Molly. 1992. Indefinites. Linguistic Inquiry Monograph 20. Cambridge, Massachusetts: MIT Press.

Diesing, Molly and E. Jelinek. 1993. The syntax and semantics of object shift. Working Papers in Scandinavian Syntax 51.

Diesing, Molly and E. Jelinek. 1994. Distributing Arguments. Ms. Cornell University and The University of Arizona.

Georgopoulos, Carol. 1991. Syntactic variables: resumptive pronouns and A' binding in Palauan. Dordrecht: Kluwer Academic Publishers.

Georgopoulos, Carol. 1992. Another look at object agreement. Proceedings of NELS 22, 165-177. GLSA, University of Massachusetts, Amherst.

Givon, Talmy. 1976. Topic, pronoun, and grammatical agreement. In Subject and topic, ed. Charles Li. New York: Academic Press.

Grimshaw, Jane. 1994. Projection, heads, and optimality. ms. Rutgers University. To appear in Linguistic Inquiry.

de Hoop, Helen. 1989. Case assignment and generalized quantifiers. Proceedings of NELS 19, 176-190. GLSA, University of Massachusetts, Amherst.

de Hoop, Helen. 1992. Case configuration and noun phrase interpretation. Doctoral dissertation, Rijksuniversiteit Groningen.

Itô, Junko and Armin Mester. 1995. The core-periphery structure of the lexicon and constraints on reranking. Ms., University of California, Santa Cruz. To appear in University of Massachusetts Occasional Papers in Linguistics.

Josephs, Lewis S. 1975. Palauan reference grammar. PALI Language Texts: Micronesia. Honolulu: The University Press of Hawaii.

Kisseberth, Charles. 1993. Optimal domains: a theory of Bantu tone. A case study for Isixhosa. Talk presented at ROW 1, Rutgers University.

Lemaréchal, Alain. 1993. Pluriel, animéité et référentialité en palau. Faits de langues 2:169-177. 
Mahajan, Anoop. 1990. The A/A-bar distinction and movement theory. Doctoral dissertation, MIT, Cambridge, Massachusetts.

Prince, Alan and Paul Smolensky. 1993. Optimality theory: constraint interaction in generative grammar. RuCCS Technical Report \#2, Rutgers University Center for Cognitive Science.

Rosenthall, Samuel. 1994. Vowel/glide alternation in a theory of constraint interaction. Doctoral dissertation, University of Massachusetts, Amherst.

Wilson, Helen. 1972. The phonology and syntax of Palauan verb affixes. University of Hawaii Working Papers 4.5.

Woolford, Ellen. 1993. Symmetric and asymmetric passives. Natural Language and Linguistic Theory 11:679-728.

Woolford, Ellen. 1994. Four-way Case systems: nominative, ergative, objective, and accusative. ms., University of Massachusetts, Amherst.

Dept. of Linguistics

South College

University of Massachusetts

Amherst, MA 01003

woolford@cs.umass.edu 